\title{
Anomalous Temperature Dependence of Gas Chromatographic Retention Indices of Polar Compounds on Non-Polar Stationary Phases
}

\author{
Alexander A. Pavlovskii ${ }^{1}$, Károly Héberger ${ }^{2}$, Igor G. Zenkevich ${ }^{1, *}$ \\ ${ }^{1}$ St. Petersburg State University, Institute for Chemistry \\ Universitetsky pr., 26, St. Petersburg 198504, Russia \\ ${ }^{2}$ Research Center for Natural Sciences, Hungarian Academy of Sciences, \\ H-1117 Budapest XI, Magyar tudósok krt. 2
}

*) Corresponding author: Prof. Igor G. Zenkevich, St. Petersburg State University, Institute for Chemistry, Universitetsky pr., 26, St. Petersburg 198504, Russia

E-mail: izenkevich@mail15.com

Phone: +007-812-428-4045

Abstract $^{1}$ ${ }^{1}$ Presented at $38^{\text {th }}$ International Symposium on Capillary Chromatography, Riva del Garda, Italy, May 18-23,
2014. 
Increasing the reliability of both GC and GC-MS identification requires appropriate interlaboratory reproducibility of gas chromatographic retention indices (I). Known temperature dependence, $I(\mathrm{~T})$ is the main source of non-reproducibility of these parameters. It can be approximated with a simple linear function $I(T)$. However, since mid-1990s beginning of 2000s some examples of anomalous temperature dependence, $I(T)$, preferably for polar analytes on non-polar stationary phases were revealed independently by different authors. The effect implies the variations in the sign of the temperature coefficients $\beta=d I / d T$ for selected compounds and, hence, the appearance of the I-extrema (usually, minima).

The current work provides evidences that the character of the anomalous $I(T)$ dependences (ascending, descending, or with extrema) is strongly influenced by the amounts of analytes injected into the chromatographic column, but these anomalies appeared not to be connected directly with the mass overloading of separation systems. The physicochemical model is proposed to describe the observed anomalies of $I(T)$ dependence. This model is based on three previously known principles of chromatography, namely:

i) the dependence of partition coefficients of polar analytes between stationary and gas phases $\left(K_{\mathrm{p}}\right)$ can be approximated with the well-known equation (sometimes called as twoparameter Antoine relationship), $\ln \left(K_{\mathrm{p}}\right)=a / T+b$;

ii) the polarity of the stationary phase of chromatographic column is varied as a result of its dynamic modification with polar analytes;

iii) the variations in the retention indices of polar analytes are proportional to the quantity of these polar analyte dissolved in the stationary phase.

The superposition of these objectives allows understanding both the unusual temperature dependence of retention indices, and the influence of the amounts of polar analytes injected into GC column on the parameters of this dependence.

Keywords: Gas Chromatography, Retention Indices, Temperature Dependence, Retention Anomalies, Polar Analytes, Non-Polar Phases 
INTRODUCTION

Retention indices $(\boldsymbol{I})$ are generally accepted as the most reproducible interlaboratory retention invariants originally introduced by Ervin sz. Kováts [1] and later sub-divided and described in generalized forms [2,3]. Their application makes it possible chromatographic (or chromato-spectral) identification of constituents of complex mixtures by the direct comparing of $I$-values with reference data:

$$
I=I_{\mathrm{n}}+\left(I_{\mathrm{n}+\mathrm{k}}-I_{\mathrm{n}}\right)\left[f\left(t_{\mathrm{R}, \mathrm{x}}\right)-f\left(t_{\mathrm{R}, \mathrm{n}}\right)\right] /\left[f\left(t_{\mathrm{R}, \mathrm{n}+\mathrm{k}}\right)-f\left(t_{\mathrm{R}, \mathrm{n}}\right)\right]
$$

where $t_{\mathrm{R}, \mathrm{n}}<t_{\mathrm{R}, \mathrm{x}}<t_{\mathrm{R}, \mathrm{n}+\mathrm{k}}$ are retention times of reference $n$-alkanes with a number of carbon atoms in the molecules $n$ and $n+k\left(I_{\mathrm{n}}=100 n\right)$ and the compound $(x)$ under characterization. The function $f\left(t_{\mathrm{R}}\right)$ is determined by the temperature regime of gas chromatographic analysis: $f\left(t_{\mathrm{R}}\right)=\lg \left(t_{\mathrm{R}}-t_{0}\right)$ (Kovats indices for isothermal conditions, $t_{0}$ is hold-up time), $f\left(t_{\mathrm{R}}\right)=t_{\mathrm{R}}$ (linear indices for temperature programming), or $f\left(t_{\mathrm{R}}\right)=t_{\mathrm{R}}+q \log \left(t_{\mathrm{R}}\right)(q \neq$ const) so-called linear-logarithmic indices for any regimes.

The $I$ values on standard non-polar (polydimethyl siloxanes) and polar (polyethylene glycols) stationary phases are known at present for more than 70 thousand organic compounds [4]. The unambiguousness of identification using single-dimensional analytical parameters (including chromatographic retention indices) strongly depends on their interlaboratory reproducibility. The lesser are standard deviations of averaged $I$-values, the lesser is the unambiguousness of the results. Hence, revealing the factors determining the data scattering and, if possible, minimization of their influence, is an important problem in chromatography.

One of the principal sources of $I$ scatter is their temperature dependence. For most organic compounds in the typical conditions of gas chromatographic analysis temperature dependence is a monotonous function, which can be approximated with linear regression that is with two first members of its expansion by Taylor's series [2, 5-7]:

$$
I(T)=I\left(T_{0}\right)+d I / d T\left(T-T_{0}\right)
$$

where $\beta=d I / d T \approx\left[I\left(T_{2}\right)-I\left(T_{1}\right)\right] /\left(T_{2}-T_{1}\right)$ is the temperature coefficient of retention indices (it obeys the inequality $\beta>0$ for most organic compounds), $T$ is a temperature of experimental measuring of retention indices, $T_{0}$ is a temperature selected as a standard value.

The values of coefficient $\beta$ at the same stationary phase in a GC column depend on the chemical structure of analytes. One of the most expressed factors is the topological connectivity of molecules (firstly, the number of cycles) [6, 7]. Known attempts of more detailed classification of parameter $\beta$ values appeared to be useless because of their low interlaboratory reproducibility.

Equation (2) is equivalent to a simple linear approximation [5-8]:

$$
I(T)=a+b T
$$

However, such approximation does not express the inherent non-linearity in the $I(T)$ data.

Therefore, more complex relations are also suggested, like the three-parameter Antoine type equation [9]:

$$
I(T)=a+b /(T+c)
$$

and a four-parametric hyperbolic relation [10]: 


$$
I(T)=(a+b T) /(1+c T)
$$

Relations (3) and (4) are monotonous functions indicating no $I(T)$ extrema. The sole nonmonotonous equation proposed by Heberger and coauthors and explaining the existence of extrema (see below) is a combined logarithmic-hyperbolic function as derived from the integration of molar heat capacities (if the latter were approximated with a polynomial) [1112]:

$$
I(T)=a+b / T+c \ln T
$$

An appearance of the minima on $I$ vs. $T$ curves was first experimentally found at mid1990s by Hennig and Engewald for such polar compounds as monoterpene alcohols and phenols (e.g., citronellol, thymol, carvacrol, etc.) [13]. Later, at the beginning of 2000s, the number of examples of anomalous $I(T)$ dependence for polar analytes on non-polar phases was increased. Such anomalies were observed for nitroalkanes and alkanenitriles [10], aldehydes and ketones [11, 12], diketones [14], $\alpha$-pinene [15], etc. It was found that reducing the temperature of GC column results first in the normal reduction of the retention indices (that corresponds to the condition $\beta>0$ ), but below some $T$-value (typically $T<100$ ${ }^{\circ} \mathrm{C}$ ) the descending $I(T)$ character is changed to ascending. It is equivalent to changing the sign of the temperature coefficients $\beta=d I / d T$ depending on temperature. As a result the plots of the dependences $I(T)$ exhibit a minimum like it is schematically shown in Fig. 1 in generalized form uniting all previously published data [11-15].

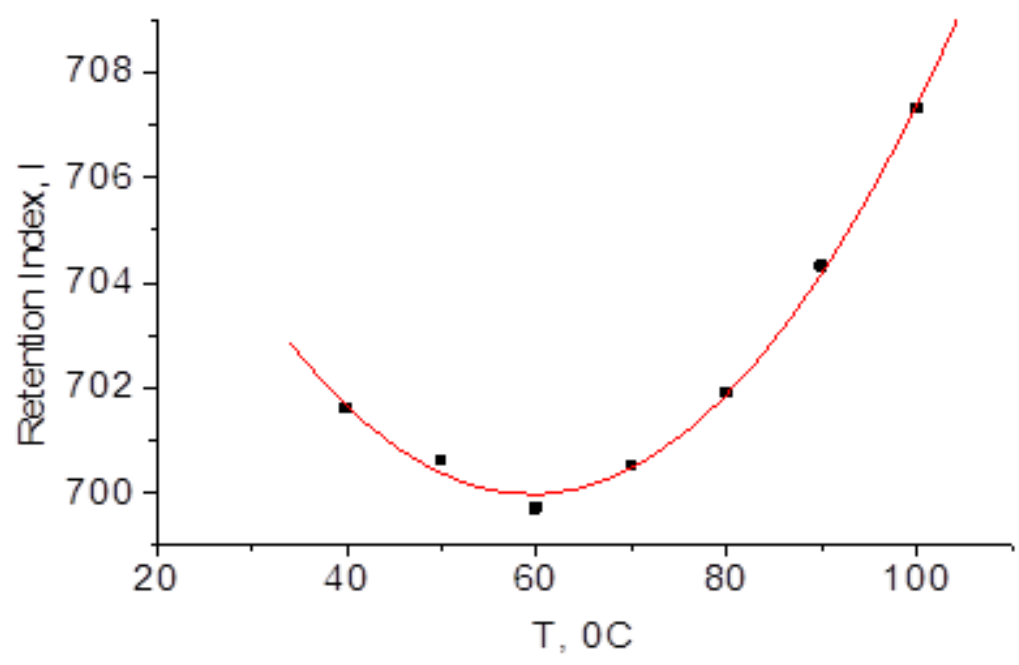

Fig. 1. Schematic plot of an anomalous dependence of retention indices of polar analytes $v s$. temperature for non-polar phases.

Santiuste and coworkers investigated in detail the temperature dependence of Kováts indices for various compounds of different classes [16-19]. They characterized 56 solutes of varied polarity on five poly-(3,3,3-trifluoropropyl)methylsiloxanes at four temperatures and found that though linear temperature dependence is significant (in the statistical sense), the use of the extended relation (Eq. 6) improves the fit [16]. Later they characterized nalkylbenzenes $\mathrm{C}_{6}-\mathrm{C}_{10}$ at isothermal conditions (from 323.15 to $423.15 \mathrm{~K}$ in increments of 10 K) [18]. Linear relationships were confirmed, but for the simplest homologues slight 
curvature could be observed in the relationships $I$ vs. T. Hence, at the nowadays comprehension of the problem is the extended model (Eq. 6) better describes all types of $I(T)$ variation with the column temperature than the Antoine type or linear relationship [17].

Three different behaviors observed [18] for structurally different solutes on a non-polar polydimethylsiloxane capillary column were considered: in the 333-423 K range. Three trends of $I$ vs. $T$ were perfectly described by the extended model based on Eq. (6). It was confirmed that, as a general rule, $I$ increases with increasing temperature, but the opposite trend was found for linear alcohols and esters. Cyclobutanol, and simplest alkylamines (1butanamine and 1-pentanamine) show well-defined I minima between 358 and 377 K. 1Nonanol show a curvature at higher temperatures, this effect was observed for higher homologs of 1-alkanols for the first time [18]. In a recent study the suitability of the extended equation (6) was affirmed: "The dependence of $I$ on column temperature was explored with the extended model with good results, as indicated by their statistics for the whole lot of solutes." [19].

In the paper of Görgényi [20] was first announced the appearance of not only concave, but convex $I(T)$ dependences (differing in the signs of second derivatives $d^{2} I / d T^{2}$ ). Generally, we may fix that anomalous $I(T)$ behavior was observed for the first several members of the homologous series especially for polar compounds.

Some attempts to theoretical interpretation of this effect $[12,16,21]$ appeared to be inconclusive just the quality of the approximation of the $I(T)$ minima to Eq. (6) were considered, but no attribution to physicochemical grounds of chromatography was performed. However, these $I(T)$ anomalies reflect the important regularities of chromatographic processes (probably, not only in gas chromatography) and, hence, deserve a detailed consideration. Similar to the "normal" $I(T)$ dependence, these anomalies determine the level of interlaboratory scattering of retention indices.

The subject of this paper is the development and discussion of the physicochemical model for the interpretation of the anomalies in $I(T)$ dependences, namely of two interrelated effects: i) the variations in the signs of coefficients $\beta=d I / d T$, and ii) the influence of the injected amounts of analytes on the positions of $I(T)$ minima and the general character of these dependences.

\section{EXPERIMENTAL}

The following compounds were selected for observing the anomalies of the $I(T)$ dependence: dimethyl formamide (DMFA), 1-butanol, dimethyl sulfoxide (DMSO), dimethyl acetamide (DMAA), 1-butanol, 1-nitropropane, and toluene (all provided by Reakhim, Moscow, Russia). The samples of tested compounds (from 10 to $250 \mu \mathrm{L}$ ) were mixed with reference $n$-alkanes $\mathrm{C}_{6}-\mathrm{C}_{8}(50-100 \mu \mathrm{L})$ or $\mathrm{C}_{6}-\mathrm{C}_{9}$ (for dimethyl sulfoxide) and these mixtures were dissolved in $0.5-5.0 \mathrm{~mL}$ of 2-propanol (Vekton, St. Petersburg, Russia). The choice of 2-propanol was due to its relatively high normal boiling point $\left(T_{\mathrm{b}} 82.4{ }^{\circ} \mathrm{C}\right)$ combined with high heat of evaporation $(0.76 \mathrm{~kJ} / \mathrm{g}$, that is twice more than that of for $\mathrm{n}$-hexane, $0.37 \mathrm{~kJ} / \mathrm{mol})$ and a low and low retention index on standard non-polar polydimethyl siloxane stationary phases $(486 \pm 9)$. Hence the peak of this solvent should not interfere with the chromatographic peak of $n$-hexane, which is required for the determination of retention indices within the range 600-800. The concentrations of all components of solutions were expressed in mass-volume units $(\mu \mathrm{g} / \mu \mathrm{L})$ using their relative densities at the ambient temperature. 
The polarity characteristics for five compounds previously shown to have the anomalies of $I(T)$ dependence, supplemented with the data for dimethyl formamide: 1-butanol, dimethyl sulfoxide, dimethyl acetamide, 1-nitropropane, and low-polar toluene are presented in Table 1 . The set of polarity characteristics was selected in accordance with the recommendations of ref. [22] and includes dielectric permeability $(\varepsilon)$, dipole moment $(\mu)$, logarithm of the partition coefficient in the standard 1-octanol/water system $(\log P)$, and the differences of GC retention indices on standard polar polyethylene glycols and non-polar polydimethyl siloxane stationary phases $\left(\Delta I=I_{\text {polar }}-I_{\text {non-polar }}\right)$.

\section{Table 1 (see at the end)}

The analyses of test-samples were carried out using gas chromatograph Krystall 5000.2 (ChromaTech, Yoshkar-Ola, Russia) equipped with a flame ionization detector and fused quartz megabore WCOT column (SGE Analytical Science) of $10 \mathrm{~m}$ length, internal diameter $0.53 \mathrm{~mm}$, and film thickness $2.65 \mu \mathrm{m}$ with standard non-polar stationary phase BPX-1 at the isothermal conditions within the temperature range $30-120{ }^{\circ} \mathrm{C}$. Carrier gas (nitrogen) flow rate was $5.1 \mu \mathrm{L} / \mathrm{min}$ (linear velocity $48.5 \mathrm{~cm} / \mathrm{s}$ ), split ratio $6.4: 1$, injector and detector temperatures were $120^{\circ} \mathrm{C}$ and $150^{\circ} \mathrm{C}$, injected amounts of all samples were $0.5-1.0 \mu \mathrm{L}$.

The evaluation of modified asymmetry factors $A^{\prime}$ (the ratio of the areas of two parts of chromatographic peaks separated by perpendiculars between peak maxima and baseline) [23] was provided using standard software "ChromaTech Analytic 2.6" (options "merged peaks" and "separate peaks"). It is different from traditional definition of asymmetry measured by ratio of two parts of the peak width measured at the level of $10 \%$ of its height, separated by the same perpendicular, $A=L_{2} / L_{1}$ [24]. The values $A$ and $A^{\prime}$ well correlate with each other, but the second indicates no dependence on the height level $(5,10$, or $50 \%)$ selected for measurements and, hence, it is a more objective criterion of the peak asymmetry.

The values of hold-up time $\left(t_{0}\right)$ were calculated using retention times of three consecutive $n$-alkanes $\mathrm{C}_{6}-\mathrm{C}_{8}$ by the method of Peterson and Hirsch modified for using recurrent dependences [25]. The logarithmic (Kováts) retention indices at isothermal conditions were calculated using QBasic program. At each temperatures 2-4 I values were determined, followed by their averaging; all $I$ values are considered to one decimal digit. The calculation of the parameters of regression equations and plotting of $I(T)$ dependences was carried out using Origin software (OriginLab Corp., USA; versions 4.1 and 8.1).

\section{RESULTS AND DISCUSSION}

The discussion of the variations in the signs of coefficients $\beta=d I / d T$ implies the evaluating the influence of the injected amounts of solutes on a character of $I(T)$ dependences. First, it seems necessary for unambiguous exclusion of any suspects to explain $I(T)$ anomalies by a possible overloading the chromatographic columns. Secondly, such a discussion is eesential, because very restricted data are available on the amounts of solutes injected into chromatographic columns in the most of publications of mid-1990s - beginning 2000s describing the anomalies of $I(T)$ dependencies [10-15]; all experiments are likely to have been fulfilled at constant injected amounts of test-compounds. The strong influence of this factor on $I(T)$ anomalies was reported first in the short communication of Zenkevich and Pavlovskii [26].

\section{Selection of polar test-compounds}


The effect of changing the signs of temperature coefficients of gas chromatographic retention indices, $\beta=d I / d T$, or, in other words, the conversion of monotonous increasing (rarely, decreasing) dependences $I(T)$ into the dependences possessing extrema (usually, minima), was reported not for any polar compounds, but only for selected members of different chemical classes [11-19]. At present we still have no general rules to pre-select organic compounds or conditions of gas chromatographic analysis indicating such anomalies. In our work we have selected five polar compounds [dimethyl formamide, 1butanol, dimethyl sulfoxide, dimethyl acetamide, 1-nitropropane, compared with low-polar hydrocarbon (toluene)] not included into the lists of polar compounds mentioned in the literature [11-19]. Table 1 (see above) unites polarity data both for some test-compounds mentioned in the literature [11-19], and solutes selected in the current work.

The values $\varepsilon, \mu, \log P$, and $\Delta I$ for DMFA, DMAA, and DMSO are close to the values of the same properties for $\mathrm{CH}_{3} \mathrm{NO}_{2}$ and $\mathrm{CH}_{3} \mathrm{CN}$ than to those of less polar carbonyl compounds (acetone and butanal). This fact allowed expecting the manifestation of anomalies of dependences $I(T)$ for such analytes. However, in spite of literature data [11-19], we have characterized dependences $I(T)$ injecting different amounts of test-compounds (by variations of the concentrations of solutes in the solutions). All the I-data for six analytes within the temperature range $30-120^{\circ} \mathrm{C}$ are presented in Table 2 .

Table 2 (see at the end)

The principal issue for interpretation of these data is the limit of mass overloading of megabore WCOT column used in our experiments determined preliminary [27]; it appears to be equal $17 \pm 4 \mu \mathrm{g}$ in chromatographic zones for test compounds of different polarity. Hence, injected amounts of all solutes not exceeded this overloading limit, but in two cases were close to it (17 $\mu \mathrm{g}$ of DMFA, and $15 \mu \mathrm{g}$ of 1-nitropropane).

For all retention indices in Table 2 the values of asymmetry factor $\left(A^{\prime}\right)$ of corresponding chromatographic peaks are indicated in parantheses.

Influence of amounts of solutes in chromatographic zones on the character of the temperature dependence of gas chromatographic retention indices

Four out of six solutes in Table 2 indicate no anomalies in $I(T)$ dependences. Three of them (toluene, DMSO, and DMAA) are characterized with ascending $I(T)$ dependences $(d I / d T>0)$ for any injected amounts of test-components, while 1-butanol, with-descending one $(d I / d T<0)$. The low-polar toluene is characterized with practically linear ascending $I(T)$ relation, which can be approximated by linear regression (Fig. 2a). For medium-polar butanol such non-linear dependence is ascending. If a minimum of this dependence exists, it should be located out of the temperature range presented on the plot, i.e., at temperatures not less than $110-120^{\circ} \mathrm{C}$ (Fig. 2b). Such variations in the character of $I(T)$ dependences are considered to be typical for organic compounds of different polarity. 
(a)

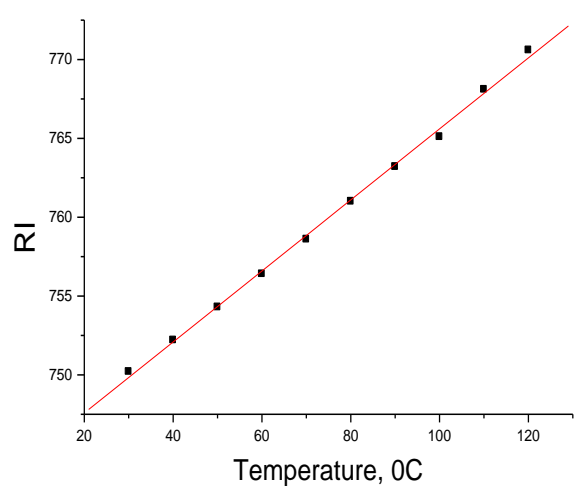

(b)

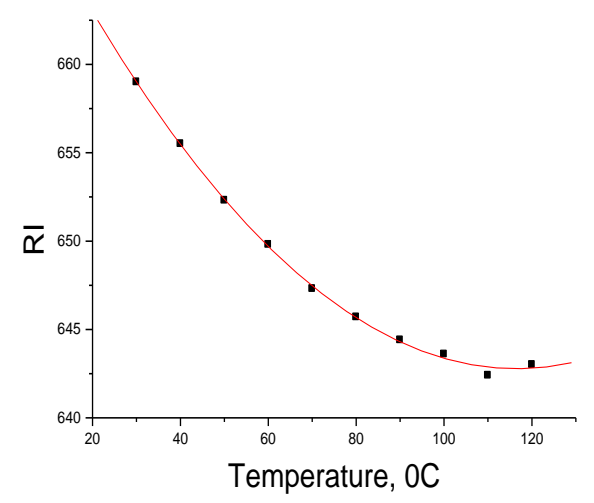

(c)

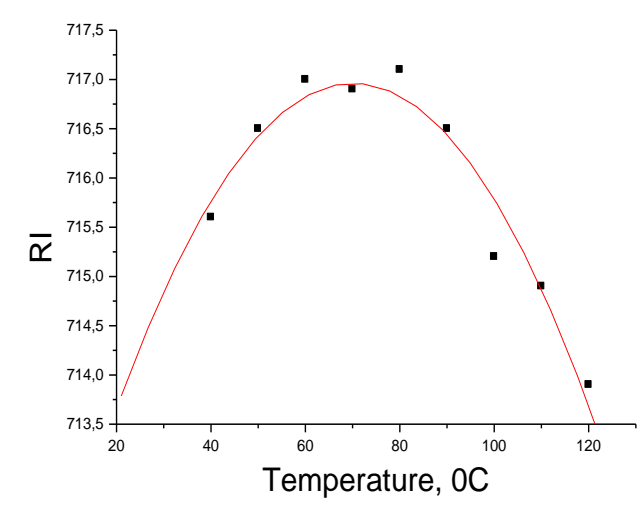

Fig. 2. Some different types of $I(T)$ dependences for test-compounds listed in Table 2: (a) linear ascending dependence $I(T)$ for low-polar toluene (amount injected $3.9 \mu \mathrm{g}$ ); (b) Non-linear descending dependence for medium-polar 1-butanol (amount injected 2.0 $\mu \mathrm{g}$ ); (c) Non-linear dependence (convex) for high polar 1-nitropropane (amount injected $15 \mu \mathrm{g})$.

Besides that, this set of data includes two examples of unusual $I(T)$ dependences. If the injected amount of 1-nitropropane is $4.5 \mu \mathrm{g}$, the "normal" ascending $I(T)$ dependence is observed. However, if the amount of this analyte is increased up to $15 \mu \mathrm{g}$, the maximum $I(T)$ is appeared within temperature range approx. $60-80{ }^{\circ} \mathrm{C}$. It is the compelling example of the non-linear convex $I(T)$ dependence (Fig. 2c).

Another compound in Table 2 (DMFA) indicates another example of the unusual $I(T)$ dependence, as it was pointed out first in the short communication [26]. Low amounts of DMFA $(0.6 \mu \mathrm{g})$ injected into chromatographic column are characterized with "normal" ascending dependence $I(T)$ without any anomalies. When this amount is close to the limit of the column mass overloading $(\sim 17 \mu \mathrm{g})$, this dependence is converted into monotonous descending. However, the middle injected amount $(4.3 \mu \mathrm{g})$ indicates a clearly expressed minimum of $I(T)$ dependence, $I_{\min }(T)=753.4$ at the temperature $\sim 80{ }^{\circ} \mathrm{C}$. It should be noted that both this temperature and $I_{\min }$ value are not fixed for this analyte and these values change at the variations of injected amount and parameters of GC column. Such minima are not observed either for the next homologue of the same class of amides (DMAA), or for DMSO in spite of close values of their polarity measures.

The revealed differences in the character of the $I(T)$ dependences on the example of DMFA seems to be so important that these data in addition to Fig. 2 should be illustrated by graphical plotting presented in Fig. 3 (a, b, c). 

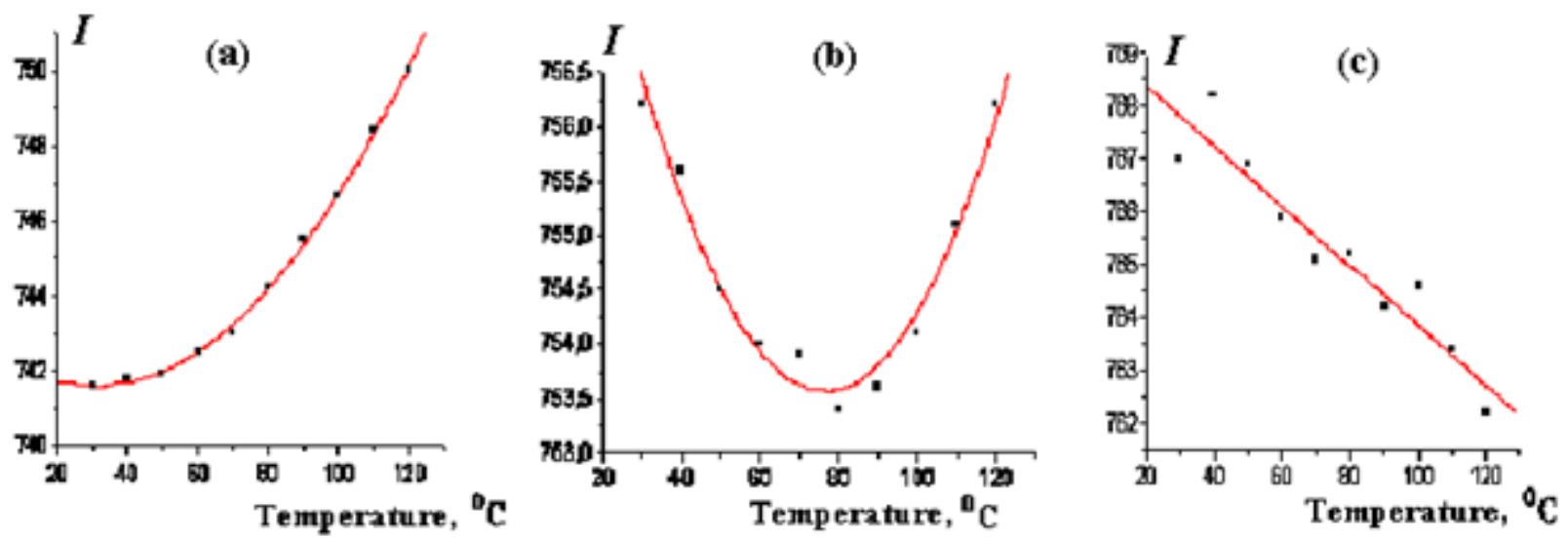

Fig. 3. Graphical presentation of data from Table 2 for dependence $I(T)$ for different injected amounts of DMFA: $0.6 \mu \mathrm{g}$ (I), $4.3 \mu \mathrm{g}$ (II) and $16.9 \mu \mathrm{g}$ (III). Solid lines correspond to $I(T)$ approximations with second degree polynomials (from [27] with permission)

This anomaly (conversion of ascending $I(T)$ dependence into descending through an intermediate case indicating a minimum) is not caused directly by mass overloading of gas chromatographic system, because the variations in the sign of $\beta=d I / d T$ are mostly expressed for injected amount of solute $4.3 \mu \mathrm{g}$ that is few times less than limiting value $17 \pm 4 \mu \mathrm{g}$ [27].

Strong dependence of the signs and absolute values of the coefficients $\beta$ on the amounts of analytes injected into chromatographic column appeared to be one of the main reasons of the low interlaboratory reproducibility of these temperature coefficients as mentioned in the Introduction. It is caused by measuring these parameters without precise controlling and reproducing the injected amounts of analytes.

Generally, $I$ vs. $T$ curves (Table 2 and Fig. 3) can be approximated with Eq. (6) $[11,12]$ fairly precisely. Besides that non-linear $I(T)$ dependences can be approximated using polynomials including the simplest second order polynomials [26], with fairly acceptable precision (the solid lines at these plots). This procedure (polynomial approximation) can be used for testing the results of the theoretical modeling of $I(\mathrm{~T})$ anomalies (see below).

The values of the asymmetry factor $\left(A^{\prime}\right)$ presented in Table 2 indicate significant variations. At high temperatures $\left(100-120^{\circ} \mathrm{C}\right)$ for most of analytes $A^{\prime}>1$ that corresponds to the little "tailing" of their chromatographic peaks. Decreasing the temperature of chromatographic separation leads to a decrease of $A^{\prime}$-values for all solutes despite of the signs of $\beta$. It is equivalent to the preferable distortions of front edges of chromatographic peaks at lower temperatures.

The increase in the injected amounts results in increased data scattering that is reflected by a decrease in correlation coefficients $(r)$. Additionally, it should be stressed that values of coefficient $A$ in equations (3), (4), and (6) increase, when the injected amount is increased: $742.7 \pm 0.3$ (a), $761.4 \pm 0.5$ (b), and $769.5 \pm 0.5$ (c). These coefficients are equal to the $I$ values at the conventional temperature $0{ }^{\circ} \mathrm{C}$. A theoretical model selected for the explanation of $I(T)$ anomalies should also explain the increase in these coefficients (see below).

Thus, we can conclude that anomalous temperature dependence of retention indices of polar compounds on non-polar phases known from literature [10-19] can be considered as partial case of a more general $I(T)$ dependence. The parameters of $I(T)$ relationships 
demonstrate the strong influence of the injected amount of analytes. Specific features of $I(T)$ dependence (appearances of minima) are observed only for a limited range of injected amounts and parameters of chromatographic columns (Fig. 2), while their variations can result in changing the sign of $\beta=d I / d T$ coefficients. Hence, among various known recommendations to approximating the dependence $I(T)$, only the Eq.(6) $[11,12]$ is appropriate, because it describes the variable character of such dependence (non-linear monotonous decreasing, non-linear monotonous increasing and containing extremes).

On the other hand, the influence of injected amount into GC column on the character of temperature dependence of retention indices suggests that polar analytes influence the polarity of a non-polar stationary phase. The approximation both "normal" and anomalous $I(T)$ dependences with Eq. (6) necessitates the elaboration of a physicochemical model.

\section{Physicochemical interpretation of anomalies of temperature dependence of gas chromatographic retention indices}

At first, let us postulate that "normal" ascending or descending linear dependences $I(T)$ are typical for most organic compounds differ from reference $n$-alkanes. Hence, its anomalies for some polar solutes can be explained by influence of additional factors summarized with normal $I(T)$ dependence.

Decreasing the temperature of gas chromatographic analysis of polar analytes in case of non-polar stationary phases usually leads to increasing asymmetry of their peaks. To illustrate this statement, data in Table 2 are compared with the corresponding values of asymmetry $\left(A^{\prime}\right)$. The values $A^{\prime}<1$ correspond to the peaks with distorted front edges, while inequality $A^{\prime}>1$ characterizes the peaks with distorted back edges. If the values of asymmetry factor belong to the window $1.25-0.75$, such peaks looks rather symmetrical, while out of this range the peak asymmetry becomes easily visible.

For small amount of polar DMFA (0.6 $\mu \mathrm{g}$, series I) injected into the column with nonpolar stationary phase $A^{\prime}$-values for any temperatures $\left(30-120^{\circ} \mathrm{C}\right)$ are located within the range $1.17-0.76$ that corresponds to fairly symmetric peaks. However, for medium injected amounts of the same analyte decreasing the temperature leads to a significantly increased asymmetry factor (from 0.27 at $30{ }^{\circ} \mathrm{C}$ to 1.11 at $120{ }^{\circ} \mathrm{C}$ ). Further increasing the injected amounts results in stronger front-distortions of peak shape, because $A^{\prime}$-values decrease to about $0.06-0.10$ (Table 2). So far as retention indices of symmetric and highly asymmetric peaks are not directly comparable, one of the reasons of unusual $I(T)$ dependence [11-19] can be the incorrect comparison of the retention indices measured for chromatographic peaks of different asymmetry.

The variations of peak asymmetry vs. temperature can be explained by the variations in the composition of a stationary phase. The polarity of a stationary phase increase at temperature decreasing due to increasing partition coefficients $(K \mathrm{p})$ of polar analytes. This effect can be classified as "dynamic modification" of stationary phase.

Nevertheless, the above explanation should be classified as a phenomenological one, because the distortion of peak shapes is not the reason, but the consequence of solute solvent interactions, as well. The reasons of changing the sign of $\beta=d I / d T$ coefficients should be interpreted using the principal (known) regularities of chromatographic retention. Let us start with the equation describing the Gaussian shape of the "ideal" chromatographic peak, $y(x)$ [24], because some variables in this equation are used in the model discussed: 


$$
y=\left(M / t_{\mathrm{R}}\right)(N / 2 \pi)^{1 / 2} \exp \left[-(N / 2)\left(1-x / t_{\mathrm{R}}\right)^{2}\right]
$$

where $N$ is the efficiency of chromatographic system expressed in theoretical plates, $M$ is the mass of the component in the chromatographic zone, $t_{\mathrm{R}}$ is the retention time of the analyte (position of peak maximum). For further modeling (see below) let us accept $t_{\mathrm{R}}=10, N=$ 1000 , and $M=3-30$ (variable parameter). The concept of "ideal" chromatographic conditions is based on the assumption that an analyte produce no influence on the properties of the stationary phase in the chromatographic column. However, in the real conditions such "sorbent - stationary phase" influences are manifested rather frequently (at first, column mass overloading effects [27] are attributed to the dissolution of oversized amounts of analytes in a stationary phase). The ratio of concentrations of analyte in stationary and mobile phases is determined by its partition coefficient, $K_{\mathrm{p}}$ :

$$
K_{\mathrm{p}}=C_{\text {stat. phase }} / C_{\text {gas phase }}=\gamma\left(M_{\text {stat. phase }} / M_{\text {gas phase }}\right)
$$

where $\gamma$ is a volume ratio of gaseous and stationary phases.

Hence, the concentration of the analyte in a stationary phase is determined by its $K_{\mathrm{p}}$ value, while its temperature dependence $K_{\mathrm{p}}(T)$. In a simplest case this dependence can be approximated with two-parameter reciprocal equation:

$$
\ln \left(K_{\mathrm{p}}\right)=a / T+b
$$

where $a$ is the coefficient proportional to the difference of the enthalpy of analyte interaction with two phases; $b$ is the same for entropy difference, $T$ is the absolute temperature, $K$. Hence, if $M$ is a total amount of the analyte injected into a chromatographic system, its mass in a stationary phase within chromatographic zone is $M_{\text {stat.phase }}=M\left[K_{\mathrm{p}} /\left(K_{\mathrm{p}}+1\right)\right]$ (to simplify the model let us select conventionally $\gamma \equiv 1$ ). If necessary, the value of $y$ that is current intensity of chromatographic signal (equation 7) can be used instead of $M$. Let us accept additionally that the retention index of the target analyte on non-polar phase is $I$, while the retention index of the same analyte on a polar phase is $I+\Delta I$. Thus, increasing the retention index of polar compounds on the stationary phase dynamically modified by this compound should be proportional to its mass fraction in the stationary phase, namely $k \times \Delta I \times M_{\text {stat. phase }}$ or $k \times \Delta I \times$ $M\left[K_{\mathrm{p}} /\left(K_{\mathrm{p}}+1\right)\right]$. Here $k$ is the additional coefficient of proportionality including all the experimental factors (e.g., even the volume ratio, $\gamma$ ) and introduced for the convenient rescaling of the model proposed (in our case let us select $k=0.1$ ). The proportionality of $I$ values to the composition of mixed stationary phases (the result of dynamic modification), namely to the mass fractions (not to the mole or volume fractions) of components of the mixed phases has been confirmed by special experiments [28].

The effect considered seems to be more important for larger amounts of analytes (when the changes in the polarity of modified stationary phase become stronger) and at the conventionally lower temperatures of GC columns. If this effect combines with the "normal" pseudo-linear temperature dependence (equation 2), i.e., $I=I\left(T_{0}\right)+\beta\left(T-T_{0}\right)$, than summarizing all the constituents mentioned for the dependence $I(T)$ we obtain the following equation:

$$
I=I\left(T_{0}\right)+\beta\left(T-T_{0}\right)+k \times \Delta I \times M \times\left[K_{\mathrm{p}} /\left(K_{\mathrm{p}}+1\right)\right]
$$

where $K_{\mathrm{p}}=\exp (a / T+b)$. In relation to the independent variable $T$ the equation (10) is a linear-exponential relation and, hence, it has no analytical solution, but it can be solved numerically. Despite of the simplicity of the theoretical basis of the Eq. (10), the appropriate selection of values of all the variables provides the adequate modeling of all the anomalies of 
$I(T)$ dependences. The Eq. (10) expresses both linear and non-linear dependence including the existence of minima.

The best way to perceive the $I(T)$ dependence (that is like semi-validation of the model proposed) is to illustrate it graphically. Such evaluation requires the choice of any appropriate values of all parameters in equation (10). At first, if we select the values of retention times and temperatures typical for GC analysis with capillary columns, we can evaluate the values of coefficients not of the temperature dependence of partition coefficients $K \mathrm{p}$ (equation 9), but the same dependence of retention times, namely $a \sim(1-3) \times 10^{3}, b \sim(-8)-(-2)$. Thus, let us select Let us select $a=2000, b=-6$. However, if we change this selection, the general character of the results remains the same. The typical values of temperature coefficients of GC retention indices are, $\beta \approx 0.2$ [6,7], and as it was proposed above $k=0.1$. For the more visible characteristics of the model let us select; two parameters variable (three values for each of them), $\Delta I=10,100$, and 200 , and $M=3,10$, and 30 conventional mass units. In the result we should compare $3 \times 3=9$ sub-figures $(\mathrm{a}-\mathrm{i})$ presented in Fig. 4 .

(d) $\Delta I=100, M=3$

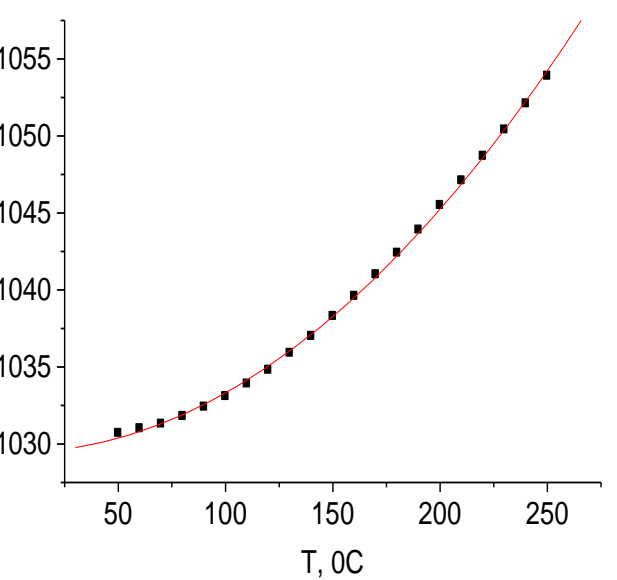

(g) $\Delta I=200, M=3$

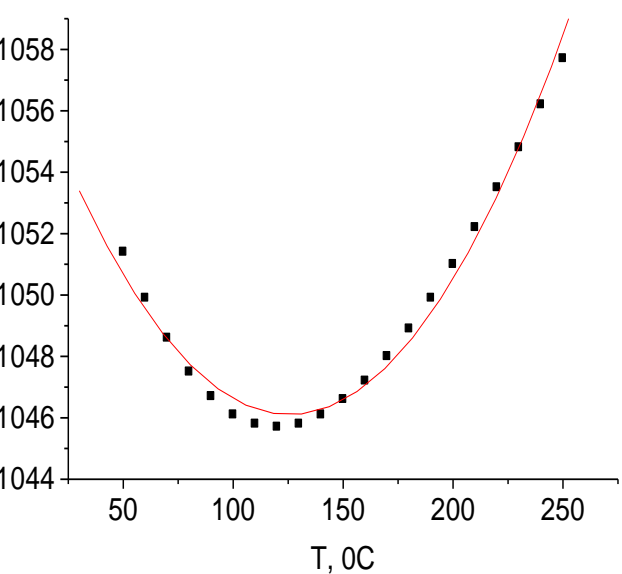

(e) $\Delta I=100, M=10$

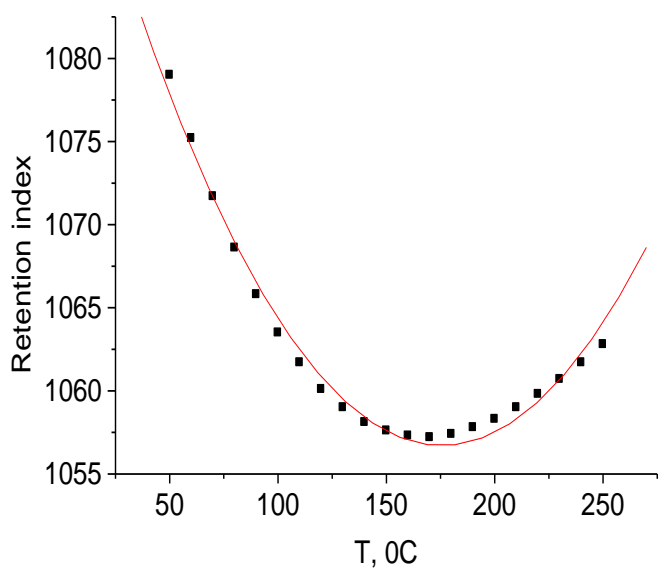

(h) $\Delta I=200, M=10$

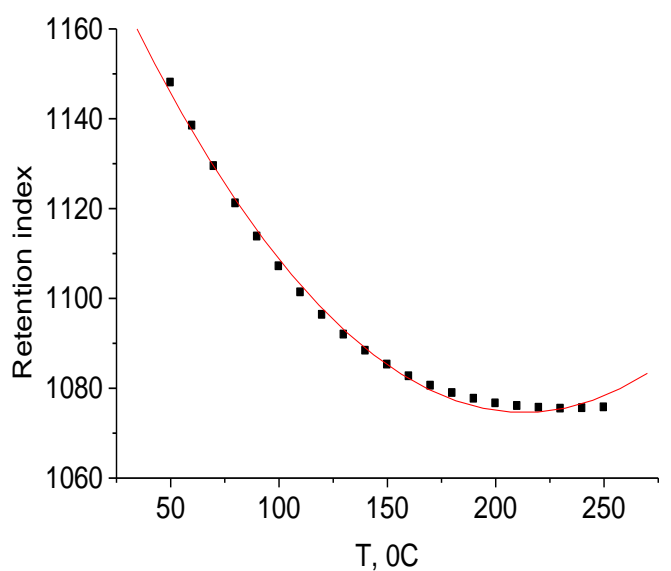

(f) $\Delta I=\mathbf{1 0 0}, M=30$

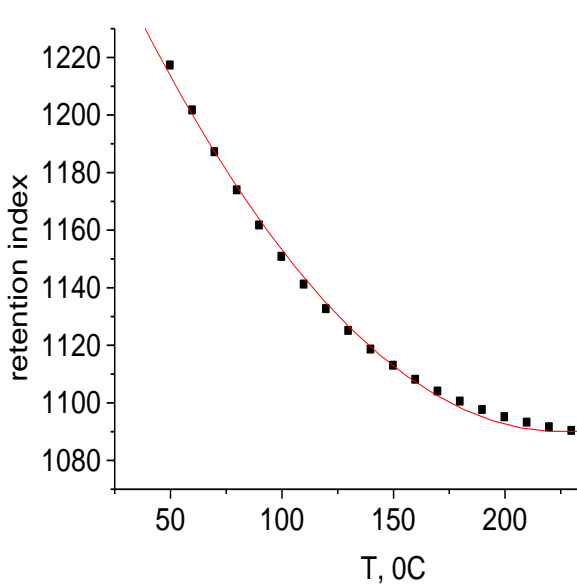

(i) $\Delta I=\mathbf{2 0 0}, M=30$

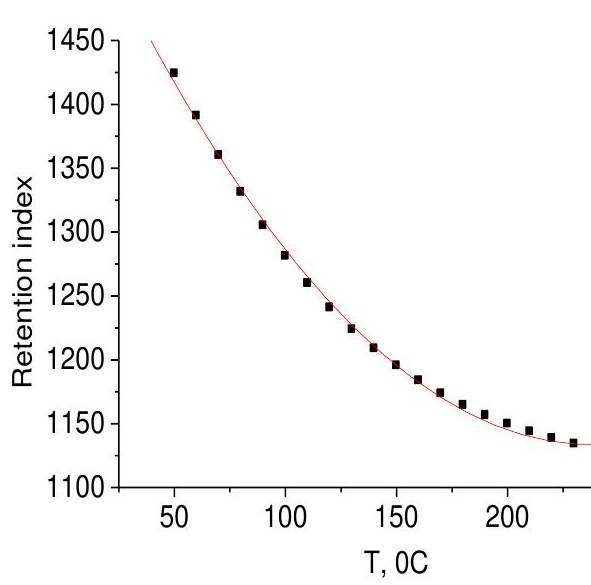


(a) $\Delta \mathrm{I}=\mathbf{1 0}, \mathrm{M}=\mathbf{3}$

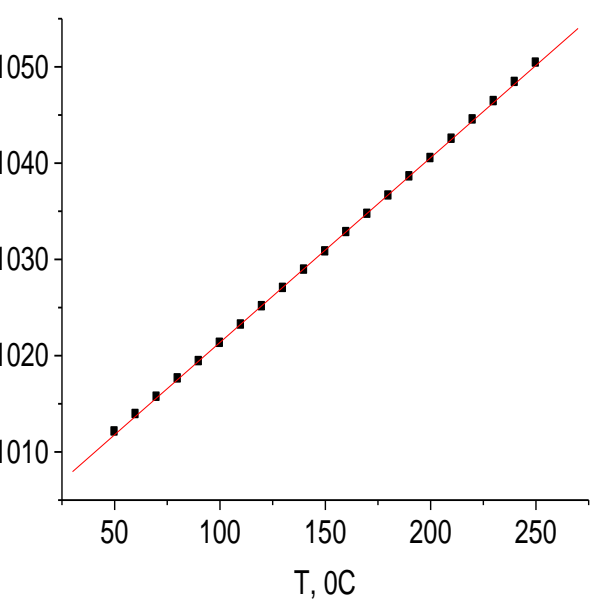

(b) $\Delta \mathrm{I}=\mathbf{1 0}, \mathrm{M}=\mathbf{1 0}$

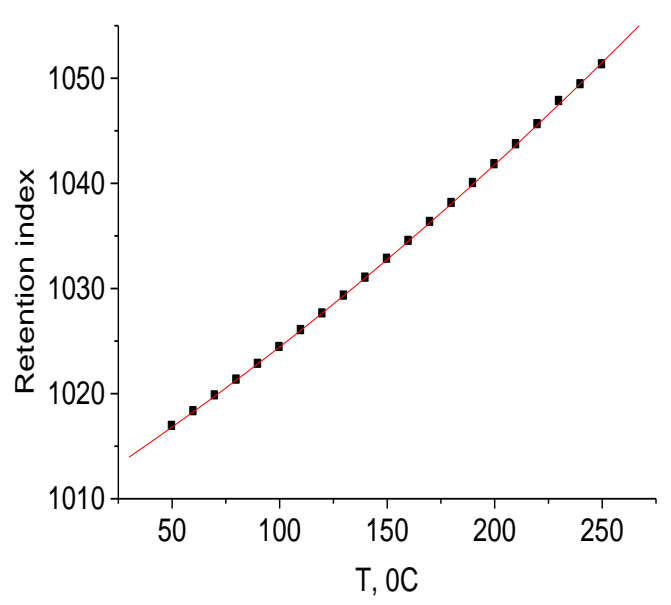

(c) $\Delta \mathrm{I}=\mathbf{1 0}, \mathrm{M}=\mathbf{3 0}$

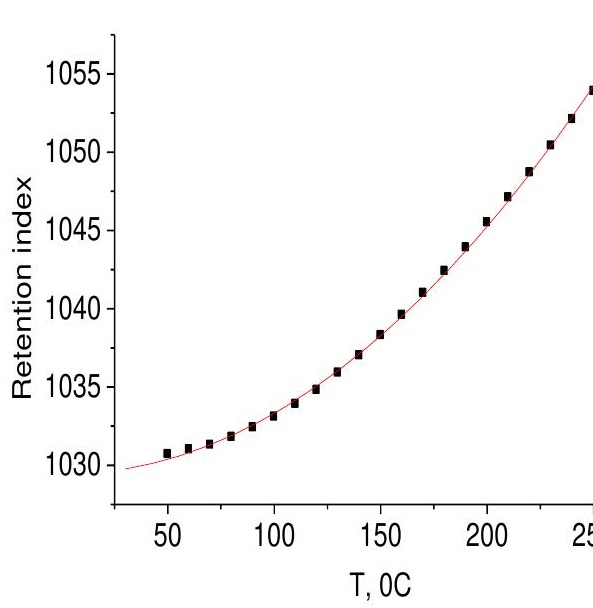

Fig. 4. The graphical presentation of the results of the numerical modeling the dependence $I(T)$ using relation (10) at the following values of parameters: $a=2000, b=-6, \beta=$ $0.2, k=0.1, \Delta I=10-200$ (variable parameter), $M=3-10$ mass units. The values $\Delta I$ and $M$ are indicated for every sub-figure (a)-(i). Solid lines correspond to the data approximations with second degree polynomials exception is the plot (a) (first order linear regression).

The smallest value $\Delta I=10$ corresponds to the analytes of minimal polarity. When the amounts of such compounds injected into chromatographic column are small $(M=3)$, we obtain the practically linear $(n=21 ; r>0.999)$ ascending dependence $I(T)$, presented in the sub-figure 4(a). An increase of amount of such non-polar analyte results in the conversion of linear dependence $I(T)$ into non-linear, as it is illustrated with Fig. 4(c).

For minimal quantities of polar analyte $(\Delta I=100, M=3)$ we obtain the non-linear ascending dependence $I(T)$ (Fig. 4d); increasing its amount up to $M=10$ results in the anomaly of the temperature dependence $I(T)$ (changing the sign of the coefficient $d I / d T$ at approximately $170{ }^{\circ} \mathrm{C}$, or, in other words, the appearance of the minimum, Fig. 4e), while further increasing the amount up to $M=30$ converts the dependence $I(T)$ into non-linear descending function (Fig. 4f).

The following increasing the polarity of analyte (up to $\Delta I=200$ ) "replace" the anomalous dependence $I(T)$ into its lesser amounts $(M=3)$ and lower temperature of the minimum $\left(T_{\min }\right.$ $\approx 110{ }^{\circ} \mathrm{C}$, Fig. $4 \mathrm{~g}$ ). Larger amounts of analytes injected are characterized with non-linear descending dependences $I(T)$, as it is shown in Fig 4(h) and 4(i). Thus, the model proposed describes the large variety of $I(\mathrm{~T})$ dependences observed.

All the experimental $I(\mathrm{~T})$ dependencies (Figs 1-3), as well as modeled plots in Fig. 4 (a-i) can be approximated polynomials, even with simplest second degree polynomial. This approximation is indicated at the all sub-figures 4 (a-i) by solid lines. The single exception is the plot in Fig. 4a, which well corresponds to the linear regression. 
The similarity of Fig. 3 (experimental data for DMFA) and Fig. 4(d), (e), and (f) (results of the calculations using equation (10) is striking. If we change the $K_{\mathrm{p}}$-value by modification of coefficients $a$ and $b$, we are able to reflect the influence of the temperature on $I(T)$ dependence.

It seems to be important that the increase of $M$ leads to an increase in the values of the coefficients $c$ (it corresponds to $I$ values at the temperature $0{ }^{\circ} \mathrm{C}$ ). That is the necessary condition of the adequacy of the physicochemical model for $I(T)$ anomalies $(\Delta I=100): I=$ $1029.5 \pm 0.3(M=3), 1049.9 \pm 0.5(M=5), 1098.0 \pm 1.0(M=10), 1196.0 \pm 3.0(M=20)$, $1293.9 \pm 3.0(M=30)$, etc. The correlation coefficients of the data sets in Fig. $3(\mathrm{a}, \mathrm{b}, \mathrm{c})$ approximated with second-order polynomials $\left(I=a T^{2}+b T+c\right)$ are $0.999(M=3, n=21)$, $0.988(M=10, n=21)$, and $0.997(M=30, n=21)$.

Decreasing $\Delta I$ value in Eq. (10), (equivalent to the separation of low-polar solutes) results in the disappearance of the minimum on the $I(T)$ temperature dependence. This is in complete accordance with the chromatographic regularities, because $I(T)$ minima were never observed for non-polar compounds analyzed with non-polar phases. However, the principal feature of the effect under consideration is its manifestation not for any polar analytes, but for some of them. Obviously, it depends on the specific chemical properties of such polar compounds, which cannot be presented adequately in the model discussed.

The ground of the $I(T)$ dependence with maxima like presented in Fig. 2c for 1-nitropropane can be explained by superposition of descending linear $I(T)$ functions with Eq. (1) considered with reciprocal signs. However, due to the relatively small number of the examples of such dependences their detailed consideration seems to be premature.

The effect of dynamic modification of sorbent in a chromatographic column is wellknown and generally accepted in high performance liquid chromatography (HPLC) [30-32], especially for chiral separations resulting additives of chiral modifiers in an eluent [33]. In gas chromatography the concept of dynamic modification of a stationary phase seems to be not so commonly accepted. It is used by necessity when chemically active carrier gases (e.g., ammonia) are used [34]. Some other effects in chromatography are caused by dynamical modification of stationary phase by large amounts of samples, as well. One of them, for example, is so-called "Solvent Effect" [29]; it is responsible for shifting the retention times of minor constituents eluted immediately after the overloaded peaks.

\section{CONCLUSIONS}

The anomalies of temperature dependence of gas chromatographic retention indices of polar analytes on non-polar phases known since the mid-1990s can be interpreted using the concept of non-ideal chromatographic processes. The reasons of such anomalies are the variations in the properties of non-polar stationary phase, modified dynamically by polar analytes during GC separation run. Such explanation is close to that of the mass overloading of chromatographic systems, but $I(T)$ anomalies are observed at the smaller amounts of analytes than overload. If necessary, just these effects can be considered as the new criterion for getting closer to the limit of overloading.

The previous descriptions of anomalous dependences $I(T)$ were based on the logarithmichyperbolic relation (6) which permits the approximation both non-linear dependences, as well as those with extrema. In contrast with this, equation (10) of the new approach is not so simple, but it allows explaining the $I(T)$ anomalies using well-known basis of chromatography. 
Thus, the principles of the model proposed for explaining the unusual temperature dependence of gas chromatographic retention indices of polar solutes on non-polar phases are the following: i) it is based on the well-known dependence of partition coefficients of polar analytes between stationary and gas phases in GC column, which can be approximated with a two-parameter reciprocal equation, $\ln \left(K_{\mathrm{p}}\right)=a / T+b$, ii) the local polarity of stationary phase in chromatographic zone of analyte is increased in the result of its dynamical modification by this analytes, and iii) the variations of retention indices of polar analytes are proportional to the mass fraction of polar analyte dissolved in the stationary phase. These objectives let understand both the unusual temperature dependence of retention indices of polar compounds on non-polar phases, and the influence of their amounts injected into GC column on the parameters of this dependence.

Additionally it seems to be important to reconsider the properties of temperature coefficients of GC retention indices. Their values are not constant, but indicate the strong dependence on amounts of analytes injected into chromatographic columns.

\section{ACKNOWLEDGEMENTS}

The experimental part of this work was fulfilled using the equipment of Educational Resource Center of Chemistry at the Institute of Chemistry at St. Petersburg State University. The authors thank the staff of this Center for helpful assistance.

\section{REFERENCES}

[1] E.sz. Kováts, Gaz-chromatographische Charakterisierung organishcher Verbindungen. Teil 1: Retentionsindices aliphatischer Halogenide, Alkohole, Aldehyde und Ketone, Helv. Chim. Acta 41 (1958) 1915-1932 [in German].

[2] R.V. Golovnya, and Yu.N. Arsen’ev, Rus. Chem. Bull., 42 (1973) 2221-2246.

[3] I.G. Zenkevich, New methods for calculation of gas chromatographic retention indices. In: "100 years of chromatography“ (in Russian). Moscow: Nauka Publ., 2003. pp. 311336.

[4] The NIST 14 Mass Spectral Library (NIST14/2014/EPA/NIH). Software/Data Version (NIST14); NIST Standard Reference Database, Number 69, 2014. National Institute of Standards and Technology, Gaithersburg, MD 20899; http://webbook.nist.gov (accessed September 2015).

[5] G.D. Mitra, and N.C. Saha, Chromatographia, 6 (1973) 93-103.

[6] C. Bangjie, and P. Shaoyi, Chromatographia, 25 (1988) 731-734.

[7] I.G. Zenkevich, In Encyclopedia of Chromatography. $3^{\text {rd }}$ Edn. Ed. J. Cazes. Boca Raton: CRC Press. 2010. P. 1304-1310.

[8] L.S. Ettre and K. Billeb, J. Chromatogr., 30 (1967) 1-11.

[9] J. Takacs, M. Rockenbauer, and J. Olacsi, J. Chromatogr., 42 (1969) 19-28.

[10] M. Gorgenyi, and K. Heberger, J. Chromatogr. A, 985 (2003)11-19.

[11] K. Heberger, M. Gorgenyi, and T. Kowalska, J. Chromatogr. A, 973 (2002) 135-142.

[12] T. Kowalska, K. Heberger, and M. Gorgenyi, Acta Chromatogr., 13 (2003) 60-68. 
[13] P. Hennig, and W. Engewald, Chromatographia, 38 (19941/2) 93-97.

[14] D.Z. Mijin, and J. Antonovic, Serb. Chem. Soc. 69 (2004) 759-767.

[15] http://rlch.hu/images/alpha-pinene.pdf (accessed September 2015).

[16] R. Lebron-Aguilar, J. E. Quintanilla-Lopez A. M. Tello, and J. M. Santiuste, J. Chromatogr. A, 1160 (2007) 276-288.

[17] J.M. Santiuste, J. E. Quintanilla-López, J. M. Takács, and R. Lebrón-Aguilar, J. Chromatogr. A, 1222 (2012) 90-97.

[18] J.M. Santiuste, J.E. Quintanilla-López, R. Becerra, and R. Lebrón-Aguilar,. Chromatogr. A, 1365 (2014) 204-211.

[19] J.M. Santiuste, J.E. Quintanilla-López, R. Becerra, C. Gutiérrez, and R. Lebrón-Aguilar, Chromatographia 78 (2015) 1071-1081.

[20] M. Gorgenyi, Z.A. Fekete, H. Van Langenhove, and J. Dewulf, J. Chromatogr. A, 1206 (2008) 180-185.

[21] K. Ciazynska-Halarewicz, E. Borucka, and T. Kowalska, Acta Chromatogr., 12 (2002) 65-79.

[22] K. Heberger, and I.G. Zenkevich, J. Chromatogr. A, 1217 (2010) 2895-2902.

[23] A.A. Makarov, and I.G. Zenkevich, Abstr. $3^{\text {rd }}$ International Symposium on Computer Application and Chemometrics in Analytical Chemistry. Budapest, Hungary. July 2006. P01.

[24] C.F. Poole, The Essence of Chromatography. Amsterdam: Elsevier, 2003, 925 p.

[25] I.G. Zenkevich, Chromatographia, 75 (2012) 767-777.

[26] I.G. Zenkevich, and A.A. Pavlovskii, Analytics \& Control, 18 (2014) 171-177 [in Russian].

[27] I.G. Zenkevich, and A.A. Pavlovskii, J. Sep. Sci., 38 (2015) 2848-2856.

[28] I.G. Zenkevich, A.A. Makarov, and K.V. Ivanova, Rus. J. Anal. Chem., 69 (2014) 10891095.

[29] K. Ciazynska-Halarewicz, and T. Kowalska, Acta Chromatogr., 13 (2003) 69-80.

[30] T. Takeuchi, and T. Mina, Chromatographia, 34 (1992) 386-390.

[31] V.A. Davankov, J. Chromatogr. A, 666 (1994) 55-76.

[32] M.G. Schmid, K. Schreiner, D. Reisinger, and G. Gubite, J. Sep. Sci., 29 (2006) 14701475 .

[33] G. Subramanian, Chiral Separation Techniques: A Practical Approach, New York: J. Wiley \& Sons, 2008. 641 p.

[34] V.G. Berezkin, V.R. Alishoev, A.A. Korolev, and I.V. Malyukova, J. Chromatogr. A, 918 (2001) 423-427.

[35] E.A.Dietz, J. High Resol. Chromatogr. 19 (1996) 485-491. 
Table 1 Physicochemical properties of some polar compounds selected for searching for the anomalies of $I(T)$ dependence

\begin{tabular}{l|c|c|c|c|c}
\hline \multicolumn{1}{c|}{ Compound } & $\begin{array}{c}\text { Molecular } \\
\text { weight, Da }\end{array}$ & $\begin{array}{c}\text { Dielectric } \\
\text { permeability, } \varepsilon\end{array}$ & $\begin{array}{c}\text { Dipole } \\
\text { moment, } \mu, \mathrm{D}\end{array}$ & $\begin{array}{c}\log P \\
I_{\text {non-polar }}\end{array}$ & $\begin{array}{c}\Delta I=I_{\text {polar }} \\
\text { Acetonitrile* }\end{array}$ \\
Propionitrile & 41 & $37.3 \pm 1.0 * *$ & $3.7 \pm 0.2$ & -0.34 & 542 \\
Acetone* & 58 & $28.1 \pm 0.8$ & $3.8 \pm 0.3$ & 0.16 & 478 \\
Nitromethane* & 61 & $37.2 \pm 1.4$ & $3.4 \pm 0.2$ & $-0.34 \pm 0.01$ & 621 \\
Butanal* & 72 & 13.4 & 2.7 & 0.88 & 299 \\
\hline Dimethyl formamide & 73 & $37.1 \pm 0.8$ & $3.8 \pm 0.1$ & -1.01 & 576 \\
1-Butanol & 74 & $17.5 \pm 0.3$ & $1.68 \pm 0.05$ & $0.87 \pm 0.02$ & 516 \\
Dimethyl sulfoxide & 78 & $47.1 \pm 1.2$ & $4.0 \pm 0.1$ & -1.35 & 779 \\
Dimethyl acetamide & 87 & 37.8 & 3.8 & -0.77 & 573 \\
1-Nitropropane & 89 & 23.2 & $3.4 \pm 0.2$ & 0.87 & 507 \\
\hline Toluene (low-polar hydrocarbon for comparison) & 92 & $2.39 \pm 0.02$ & $0.36 \pm 0.04$ & $2.74 \pm 0.06$ & 290 \\
\hline
\end{tabular}

*) Test-compounds characterized in the literature (for comparison);

**) Here and hereinafter the standard deviations of averaged literature values are indicated after the symbol «t». 
Table 2 Variations of retention indices and peak asymmetry of different polar compounds on non-polar phase caused by the variation of their injected amounts and column temperature

\begin{tabular}{|c|c|c|c|c|c|c|c|}
\hline Solute & \multicolumn{3}{|c|}{ Dimethyl formamide } & \multicolumn{4}{|c|}{ 1-Butanol } \\
\hline $\begin{array}{l}\text { Injected } \\
\text { amount, } \mu \mathrm{g}\end{array}$ & 0.6 & 4.3 & 17 & 0.2 & 0.7 & 2.0 & 6.1 \\
\hline$T,{ }^{\circ} \mathrm{C}$ & $I\left(A^{\prime}\right)$ & $I\left(A^{\prime}\right)$ & $I\left(A^{\prime}\right)$ & $I\left(A^{\prime}\right)$ & $I\left(A^{\prime}\right)$ & $I\left(A^{\prime}\right)$ & $I\left(A^{\prime}\right)$ \\
\hline 120 & $750.0(1.17)$ & $756.2(1.10)$ & $762.2(0.64)$ & $643.2(1.37)$ & $643.2(1.39)$ & $643.0(1.62)$ & $642.2(1.89)$ \\
\hline 110 & 748.4 (1.07) & $755.1(1.01)$ & $763.4(0.35)$ & $643.2(1.45)$ & - & $642.4(1.31)$ & $642.5(1.40)$ \\
\hline 100 & $746.7(1.03)$ & $754.1(0.69)$ & $764.6(0.22)$ & $643.3(1.34)$ & $643.3(1.29)$ & $643.6(1.23)$ & $643.7(1.41)$ \\
\hline 90 & 745.5 (0.99) & $753.6(0.56)$ & $764.2(0.17)$ & $644.1(1.18)$ & - & 644.4.(1.17) & $645.2(1.08)$ \\
\hline 80 & $744.2(0.94)$ & $753.4(0.47)$ & $765.2(0.14)$ & - & $644.9(1.16)$ & & $647.1(0.83)$ \\
\hline 70 & $743.0(0.76)$ & $753.9(0.39)$ & $765.1(0.09)$ & $646.0(1.07)$ & - & $647.3(0.90)$ & $649.2(0.64)$ \\
\hline 60 & $742.5(0.78)$ & $754.0(0.33)$ & $765.9(0.10)$ & - & $648.3(0.98)$ & $649.8(0.75)$ & $652.3(0.45)$ \\
\hline 50 & $741.9(0.76)$ & $754.5(.28)$ & $766.9(0.12)$ & $649.9(1.10)$ & - & $652.3(0.64)$ & $656.0(0.32)$ \\
\hline 40 & $741.8(0.77)$ & $755.6(0.27)$ & $768.2(0.06)$ & - & $653.1(0.92)$ & $655.5(0.50)$ & $658.0(0.31)$ \\
\hline 30 & $741.6(0.83)$ & $756.2(0.32)$ & $767.0(0.11)$ & $655.9(1.02)$ & - & $659.0(0.42)$ & $661.8(0.22)$ \\
\hline $\begin{array}{l}\text { Character of } \\
I(T) \\
\text { dependence }\end{array}$ & ascending & $\begin{array}{c}\text { anomalous } \\
\text { with minimum }\end{array}$ & descending & descending & descending & descending & descending \\
\hline$C$ in Eq. (5) & $742.7 \pm 0.3$ & $761.4 \pm 0.5$ & $769.5 \pm 0.5$ & $667.2 \pm 0.6$ & $667.7 \pm 0.3$ & $672.2 \pm 0.5$ & $675.3 \pm 1.0$ \\
\hline
\end{tabular}


Table 2 Continued

\begin{tabular}{|c|c|c|c|c|c|c|c|}
\hline Solute & \multicolumn{2}{|c|}{ Dimethyl sulfoxide } & \multicolumn{2}{|c|}{ Dimethyl acetamide } & \multicolumn{2}{|c|}{ 1-Nitropropane } & Toluene \\
\hline $\begin{array}{l}\text { Injected } \\
\text { amount, } \mu \mathrm{g}\end{array}$ & 1.1 & 5.0 & 4.0 & 11 & 4.5 & 15 & 3.9 \\
\hline$T,{ }^{\circ} \mathrm{C}$ & $I\left(A^{\prime}\right)$ & $I\left(A^{\prime}\right)$ & $I\left(A^{\prime}\right)$ & $I\left(A^{\prime}\right)$ & $I\left(A^{\prime}\right)$ & $I\left(A^{\prime}\right)$ & $I\left(A^{\prime}\right)$ \\
\hline 120 & $796.0(0.92)$ & $800.2(0.47)$ & $842.0(1.03)$ & $843.9(0.52)$ & $713.5(1.08)$ & $713.9(1.22)$ & $770.6(1.33)$ \\
\hline 110 & $793.3(0.80)$ & $798.2(0.37)$ & $839.8(0.69)$ & $842.9(0.41)$ & $713.0(0.98)$ & $714.9(0.80)$ & $768.1(1.20)$ \\
\hline 100 & $790.8(0.68)$ & $796.0(0.39)$ & $838.2(0.81)$ & $842.7(0.32)$ & - & $715.2(0.53)$ & $765.6(1.15)$ \\
\hline 90 & $788.8(0.63)$ & $794.4(0.24)$ & $837.3(0.49)$ & $841.5(0.28)$ & $711.7(0.69)$ & $716.5(0.35)$ & $763.2(1.03)$ \\
\hline 80 & $787.3(0.57)$ & $793.2(0.21)$ & $836.4(0.60)$ & $841.0(0.25)$ & $711.0(0.64)$ & $717.1(0.27)$ & $761.0(0.97)$ \\
\hline 70 & $786.4(0.42)$ & $791.9(0.17)$ & $836.0(0.50)$ & $840.3(0.20)$ & $710.4(0.52)$ & $716.9(0.20)$ & $758.6(0.90)$ \\
\hline 60 & $785.2(0.41)$ & $791.4(0.14)$ & $835.3(0.33)$ & $839.3(0.20)$ & $710.0(0.47)$ & $717.0(0.18)$ & $756.4(0.84)$ \\
\hline 50 & $784.7(0.50)$ & $790.0(0.17)$ & $835.0(0.47)$ & $838.8(0.16)$ & $709.1(0.39)$ & $716.5(0.13)$ & $754.3(0.75)$ \\
\hline 40 & $784.6(0.88)$ & $790.0(0.26)$ & $834.2(0.36)$ & $838.0(0.18)$ & $708.8(0.32)$ & $715.6(0.15)$ & $752.2(0.76)$ \\
\hline 30 & - & - & - & $837.0(0.19)$ & - & - & $750.2(0.72)$ \\
\hline $\begin{array}{l}\text { Character } \\
\text { of } I(T) \\
\text { dependence }\end{array}$ & ascending & ascending & ascending & ascending & ascending & $\begin{array}{c}\text { anomalous } \\
\text { with maximum }\end{array}$ & ascending \\
\hline$C$ in Eq. (5) & $787.8 \pm 0.5$ & $790.6 \pm 0.8$ & $836.0 \pm 1.2$ & $834.9 \pm 0.2$ & $706.2 \pm 0.1$ & $710.5 \pm 1.0$ & $743.0 \pm 0.2 *$ \\
\hline
\end{tabular}

*) Linear regression: $\beta=0.22 \pm 0.00 ; r=0.9994 ; S_{0}=0.3$. 
